\title{
Tensor Modes from a Primordial Hagedorn Phase of String Cosmology
}

\section{Citation}

Brandenberger, Robert H., Ali Nayeri, Subodh P. Patil, and Cumrun Vafa. 2007. “Tensor Modes from a Primordial Hagedorn Phase of String Cosmology." Physical Review Letters 98 (23). https://doi.org/10.1103/physrevlett.98.231302.

\section{Permanent link}

http://nrs.harvard.edu/urn-3:HUL.InstRepos:41385000

\section{Terms of Use}

This article was downloaded from Harvard University's DASH repository, and is made available under the terms and conditions applicable to Other Posted Material, as set forth at http:// nrs.harvard.edu/urn-3:HUL.InstRepos:dash.current.terms-of-use\#LAA

\section{Share Your Story}

The Harvard community has made this article openly available.

Please share how this access benefits you. Submit a story.

Accessibility 
HUTP-06/A0012

\title{
Tensor Modes from a Primordial Hagedorn Phase of String Cosmology
}

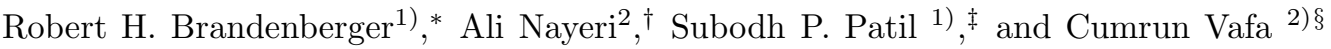 \\ 1) Dept.of Physics, McGill University, Montréal QC, H3A 2T8, Canada and \\ 2) Jefferson Physical Laboratory, Harvard University, Cambridge, MA 02138, U.S.A.
}

(Dated: October 10, 2018)

\begin{abstract}
It has recently been shown that a Hagedorn phase of string gas cosmology can provide a causal mechanism for generating a nearly scale-invariant spectrum of scalar metric fluctuations, without the need for an intervening period of de Sitter expansion. In this paper we compute the spectrum of tensor metric fluctuations (gravitational waves) in this scenario, and show that it is also nearly scale-invariant. However, whereas the spectrum of scalar modes has a small red-tilt, the spectrum of tensor modes has a small blue tilt, unlike what occurs in slow-roll inflation. This provides a possible observational way to distinguish between our cosmological scenario and conventional slowroll inflation.
\end{abstract}

PACS numbers: $98.80 . \mathrm{Cq}$

Introduction. String gas cosmology (SGC) 1, 2] (see also [3] for early work, [4, [5] for recent overviews, and [6] for a critical review) is an early approach to string cosmology, based on adding minimal but crucial inputs from string theory, namely new degrees of freedom - string oscillatory and winding modes - and new symmetries - Tduality - to the now standard hypothesis of a hot and small early universe. One key aspect of SGC is that the temperature cannot exceed a limiting temperature, the Hagedorn temperature $T_{H}$ []]. This immediately provides a qualitative reason which leads us to expect that string theory can resolve cosmological singularities [1]. From the equations of motion of string gas cosmology [2, 8] it in fact follows that if we follow the radiationdominated Friedmann-Robertson-Walker (FRW) phase of standard cosmology into the past, a smooth transition to a quasi-static Hagedorn phase will occur. In this phase, the string metric is quasi-static while the dilaton is time-dependent. Reversing the time direction in this argument, we can set up the following new cosmological scenario [1]: the universe starts out in a quasistatic Hagedorn phase during which thermal equilibrium can be established over a large scale (a scale sufficiently large for our current universe to grow out of it following the usual non-inflationary cosmological dynamics). The quasi-static phase, however, is not a stable fixed point of the dynamics, and eventually a smooth transition to a radiation-dominated FRW phase will occur, after which point the universe evolves as in standard cosmology.

Recently [9, 10] it was discovered that string thermodynamic fluctuations during the Hagedorn phase lead to scalar metric fluctuations which are adiabatic and nearly scale-invariant at late times, thus providing a simple alternative to slow-roll inflation for establishing such perturbations. It is to be emphasized that this mechanism for the generation of the primordial perturbations is intrinsically stringy - particle thermodynamic fluctuations would lead to a spectrum with a large and phenomenologically unacceptable blue tilt.
We briefly remind the reader of the key features of the new structure formation scenario. The Figure shows a sketch of the space-time evolution: at early times $t$ $\left(t<t_{R}\right)$ the universe is in the quasi-static Hagedorn phase. The physical wavelength of any perturbation mode (characterized by having constant momentum $k$ in comoving coordinates) is approximately constant. The key point is that the Hubble radius (which sets the limit on which causal processes can locally set up fluctuations - see e.g. 11] for a concise overview of the theory of cosmological perturbations and [12] for a comprehensive review) is essentially infinite, thus allowing a causal mechanism for the generation of primordial fluctuations. Near $t=t_{R}$, a smooth transition from the Hagedorn phase to the radiation-dominated phase of standard cosmology occurs. The Hubble radius decreases rapidly to take on a minimal value which is microscopic (set by the temperature at $t=t_{R}$ which will be close to the Hagedorn temperature). Thus, fluctuation modes of relevance to current cosmological observations exit the Hubble radius at times $t_{i}(k)$ close to $t_{R}$. During the radiation-dominated FRW phase, the Hubble radius increases linearly in $t$, while the physical wavelength of a perturbation mode grows only as $t^{1 / 2}$. Thus, at late times $t_{f}(k)$ the fluctuation modes will re-enter the Hubble radius. Since the primordial perturbations in our scenario are of thermal origin (and there are no non-vanishing chemical potentials), they will be adiabatic, and since they propagate on superHubble scales for a long time during the FRW phase, they will be squeezed and will lead to the same type of acoustic oscillations in the angular power spectrum of the cosmic microwave background (CMB) anisotropies as what is produced in slow-roll inflation models.

In this Letter, we generalize our previous analysis [9, 10] to allow us to compute, in addition to the scalar metric fluctuations (the metric perturbation modes which transform as scalars under spatial rotations and which couple to matter), also the tensor modes (gravitational waves). We find that the resulting spectrum of 
tensor modes is also nearly scale-invariant, but that it has a slightly blue tilt, unlike what happens in slow-roll inflation where the tilt for the gravitational wave spectrum is also red. The scalar to tensor ratio is calculable from the detailed dynamics of the system (we postpone this calculation to a followup paper). It is set by how close the temperature is to the Hagedorn temperature when the scales which are measured today exit the Hubble radius at the end of the quasi-static Hagedorn phase. It is not suppressed by the same small parameter which sets the amplitude of the spectrum of scalar metric fluctuations. Thus, a measurement of the background of stochastic gravitational waves would allow us to distinguish our scenario from the usual slow-roll inflationary models.

The outline of this Letter is as follows: we begin with a quick recap of the method of 9, 10 to compute mass fluctuations in the Hagedorn phase of SGC and generalize the method to yield fluctuations of arbitrary components of the energy-momentum tensor of the string gas. Then, we relate the spectrum of gravitational waves in late time cosmology to the fluctuations of the energymomentum tensor in the Hagedorn phase and show that the resulting power spectrum is nearly scale-invariant, like the spectrum of the scalar metric fluctuations.

In the following, we assume that our three spatial dimensions are already large during the Hagedorn phase (for a possible mechanism to achieve this see [13]), while the extra spatial dimensions are confined to the string scale. For a mechanism to achieve this in the context of SGC see [1] (see however [14, 15] for caveats), and for a natural dynamical mechanism arising from SGC to stabilize all of the moduli associated with the extra spatial dimensions see 16, 17, 18, 19, 20, 21. To be specific, we take our three dimensions to be toroidal. The existence of one cycles results in the stability of string winding modes - and this is a key ingredient in our calculations.

Energy-Momentum Tensor Correlation Functions for Closed Strings. We begin with the thermal canonical partition function $Z$ of a gas of closed strings, from which the mean energy-momentum tensor $\left\langle T^{\mu}{ }_{\nu}\right\rangle$ can be found by

$$
\left\langle T^{\mu}{ }_{\nu}\right\rangle=2 \frac{G^{\mu \alpha}}{\sqrt{-G}} \frac{\partial \ln Z}{\partial G^{\alpha \nu}} \equiv \mathcal{D}_{\nu}^{\mu} \ln Z,
$$

where $G_{\mu \nu}$ is the Euclidean metric of space-time (the time coordinate is compactified to a circle of radius $\beta$, where $\beta$ is the inverse temperature.

Our aim is to calculate the fluctuations of the energymomentum tensor on various length scales $R$. For each value of $R$, we will take our spatial coordinates to run over a fixed interval, e.g. $[0,2 \pi]$. Thus, the metric will have dimensions and will be given by

$$
G_{\mu \nu}=\operatorname{diag}\left[\beta^{2}, R^{2}, R^{2}, R^{2}\right] .
$$

Letting another derivative operator $\mathcal{D}$ act on $\ln Z$ gives two terms, one for which both derivative operators act on $Z$, a second which will contain the product of terms where one $\mathcal{D}$ acts on $Z$. Specifically (and symmetrizing over the indices), we find the mean square fluctuation to be

$$
\begin{aligned}
C_{\nu}^{\mu}{ }_{\nu}{ }_{\lambda} & =\left\langle\delta T^{\mu}{ }_{\nu} \delta T_{\lambda}^{\sigma}\right\rangle=\left\langle T^{\mu}{ }_{\nu} T^{\sigma}{ }_{\lambda}\right\rangle-\left\langle T^{\mu}{ }_{\nu}\right\rangle\left\langle T^{\sigma}{ }_{\lambda}\right\rangle \\
& =2 \frac{G^{\mu \alpha}}{\sqrt{-G}} \frac{\partial}{\partial G^{\alpha \nu}}\left(\frac{G^{\sigma \delta}}{\sqrt{-G}} \frac{\partial \ln Z}{\partial G^{\delta \lambda}}\right) \\
& +2 \frac{G^{\sigma \alpha}}{\sqrt{-G}} \frac{\partial}{\partial G^{\alpha \lambda}}\left(\frac{G^{\mu \delta}}{\sqrt{-G}} \frac{\partial \ln Z}{\partial G^{\delta \nu}}\right),
\end{aligned}
$$

with $\delta T^{\mu}{ }_{\nu}=T^{\mu}{ }_{\nu}-\left\langle T^{\mu}{ }_{\nu}\right\rangle$.

The partition function $Z=\exp (-\beta F)$ is given by the string free energy $F$. Thus, the string thermodynamical fluctuation in the energy density, here denoted by the correlation function $C^{0}{ }_{0}^{0}{ }_{0}$, becomes

$$
\begin{aligned}
C_{0}^{0}{ }_{0}^{0}{ }_{0} & =\left\langle\delta \rho^{2}\right\rangle=\left\langle\rho^{2}\right\rangle-\langle\rho\rangle^{2} \\
& =-\frac{1}{R^{6}} \frac{\partial}{\partial \beta}\left(F+\beta \frac{\partial F}{\partial \beta}\right)=\frac{T^{2}}{R^{6}} C_{V} .
\end{aligned}
$$

where $C_{V}=(\partial\langle E\rangle / \partial T)$ with $E \equiv F+\beta(\partial F / \partial \beta)$ and $V=R^{3}$ is the volume of three compact but large spatial dimensions. The fluctuation in spatial diagonal parts of the energy-momentum tensor can be found by

$$
\begin{aligned}
C^{i}{ }_{i}{ }_{i}{ }_{i} & =\left\langle\delta T^{i}{ }_{i}{ }^{2}\right\rangle=\left\langle T^{i}{ }_{i}{ }^{2}\right\rangle-\left\langle T^{i}{ }_{i}{ }^{2}\right. \\
& =\frac{1}{\beta R^{3}} \frac{\partial}{\partial \ln R}\left(-\frac{1}{R^{3}} \frac{\partial F}{\partial \ln R}\right)=\frac{1}{\beta R^{2}} \frac{\partial p}{\partial R},
\end{aligned}
$$

with no summation on $i$. The pressure $p$ is given by

$$
p \equiv-V^{-1}(\partial F / \partial \ln R)=T(\partial S / \partial V)_{E} .
$$

In the following, we will compute the two correlation functions (4) and (5) using tools from string statistical mechanics. Specifically, we will be following the discussion in [22] (see also 23, 24, 25, 26]). The starting point is the formula $S(E, R)=\ln \Omega(E, R)$ for the entropy in terms of $\Omega(E, R)$, the density of states. The density of states of a gas of closed strings on a large three-dimensional torus (with the radii of all internal dimensions at the string scale) was calculated in 22] and is given by

$$
\Omega(E, R) \simeq \beta_{H} e^{\beta_{H} E+n_{H} V}\left[1+\delta \Omega_{(1)}(E, R)\right],
$$

where $\delta \Omega_{(1)}$ comes from the contribution to the density of states (when writing the density of states as a Laplace transform of $Z(\beta)$, which involves integration over $\beta$ ) from the closest singularity point $\beta_{1}$ to $\beta_{H}=\left(1 / T_{H}\right)$ in the complex $\beta$ plane. Note that $\beta_{1}<\beta_{H}$, and $\beta_{1}$ is real. From 22 we have

$$
\delta \Omega_{(1)}(E, R)=-\frac{\left(\beta_{H} E\right)^{5}}{5 !} e^{-\left(\beta_{H}-\beta_{1}\right)\left(E-\rho_{H} V\right)} .
$$


In the above, $n_{H}$ is a (constant) number density of order $\ell_{s}^{-3}\left(\ell_{s}\right.$ being the string length) and $\rho_{H}$ is the 'Hagedorn Energy density' of the order $\ell_{s}^{-4}$, and

$$
\beta_{H}-\beta_{1} \sim\left\{\begin{array}{l}
\left(\ell_{s}^{3} / R^{2}\right), \text { for } R \gg \ell_{s} \\
\left(R^{2} / \ell_{s}\right), \text { for } R \ll \ell_{s} .
\end{array}\right.
$$

To ensure the validity of Eq. (7) we demand that $\delta \Omega_{(1)} \ll$ 1 by assuming $\rho \equiv(E / V) \gg \rho_{H}$.

Combining the above results, we find that he entropy of the string gas in the Hagedorn phase is given by

$$
S(E, R) \simeq \beta_{H} E+n_{H} V+\ln \left[1+\delta \Omega_{(1)}\right],
$$

and therefore the temperature $T(E, R) \equiv\left[(\partial S / \partial E)_{V}\right]^{-1}$ will be

$T \simeq\left(\beta_{H}+\frac{\partial \delta \Omega_{(1)} / \partial E}{1+\delta \Omega_{(1)}}\right)^{-1} \simeq T_{H}\left(1+\frac{\beta_{H}-\beta_{1}}{\beta_{H}} \delta \Omega_{(1)}\right)$.

Using this relation, we can express $\delta \Omega_{(1)}$ in terms of $T$ and $R$ via

$$
\ell_{s}^{3} \delta \Omega_{(1)} \simeq-\frac{R^{2}}{T_{H}}\left(1-\frac{T}{T_{H}}\right) .
$$

In addition, we find

$$
\langle E\rangle \simeq \ell_{s}^{-3} R^{2} \ln \left[\frac{\ell_{s}^{3} T}{R^{2}\left(1-T / T_{H}\right)}\right] .
$$

Note that to ensure that $\delta \Omega_{(1)} \ll 1$ and $\langle E\rangle \gg \rho_{H} R^{3}$, one should demand $\left(1-T / T_{H}\right) \gg\left(\ell_{s}^{2} / R^{2}\right)$.

The results (10) and (12) now allow us to compute the correlation functions (44) and (5). We first compute the energy correlation function (4), noting that

$$
C_{V} \equiv-\left[T^{2}\left(\frac{\partial^{2} S(E, R)}{\partial E^{2}}\right)_{V}\right]^{-1} \approx \frac{R^{2} / \ell^{3}}{T\left(1-T / T_{H}\right)},
$$

from which we get

$$
C^{0}{ }_{0}^{0}{ }_{0}=\left\langle\delta \rho^{2}\right\rangle \simeq \frac{T}{\ell_{s}^{3}\left(1-T / T_{H}\right)} \frac{1}{R^{4}} .
$$

Note that the factor $\left(1-T / T_{H}\right)$ in the denominator is responsible for giving the spectrum a slight red tilt. It comes from the differentiation with respect to $T$.

Evaluating (6)

$$
p(E, R) \approx n_{H} T_{H}-\frac{2}{3} \frac{\left(1-T / T_{H}\right)}{\ell_{s}^{3} R} \ln \left[\frac{\ell_{s}^{3} T}{R^{2}\left(1-T / T_{H}\right)}\right],
$$

immediately yields

$$
C^{i}{ }_{i}^{i}{ }_{i} \simeq \frac{T\left(1-T / T_{H}\right)}{\ell_{s}^{3} R^{4}} \ln ^{2}\left[\frac{R^{2}}{\ell_{s}^{2}}\left(1-T / T_{H}\right)\right] .
$$

Note that since no temperature derivative is taken, the factor $\left(1-T / T_{H}\right)$ remains in the numerator, and it is this fact which will lead to the slight blue tilt of the spectrum of gravitational waves.

Tensor Modes from Hagedorn Fluctuations. In this section we estimate the dimensionless power spectrum of gravitational waves. First, we make some general comments. In slow-roll inflation, to leading order in perturbation theory matter fluctuations do not couple of tensor modes. This is due to the fact that the matter background field is slowly evolving in time and the leading order gravitational fluctuations are linear in the matter fluctuations. In our case, the background is not evolving (at least at the level of our computations), and hence the dominant metric fluctuations are quadratic in the matter field fluctuations. At this level, matter fluctuations induce both scalar and tensor metric fluctuations. Based on this consideration we expect that in our string gas cosmology scenario, the ratio of tensor to scalar metric fluctuations will be larger than in simple slow-roll inflationary models.

We will extract the amplitude of the gravitational wave spectrum from the spatial fluctuations $C^{i}{ }_{j}{ }^{i} j$ of the energy-momentum tensor. Strictly speaking, it is the offdiagonal components which will couple uniquely to the tensor modes. We will estimate their order of magnitude by the order of magnitude of the diagonal terms computed in the previous section. This gives a good approximation, as can be checked by letting the metric in (2) depend on three separate scales $R_{i}$ (where the index $i$ runs from 1 to 3 ), and by extracting the off-diagonal correlation functions following the method of the previous section, but taking mixed spatial derivatives.

Tensor perturbations in a spatially flat FRW universe take the form

$$
d s^{2}=-d t^{2}+a^{2}(t)\left(\delta_{i j}+h_{i j}\right) d x^{i} d x^{j} .
$$

Since to linear order in $h_{i j}$ the Einstein tensor for fluctuations on a scale $k$ is proportional to $k^{2} h_{i j}(k)$, it follows from the space-space Einstein equations that

$$
k^{2} h_{i j}(k) \sim(8 \pi G) \delta T_{i j}(k) .
$$

The power spectrum of the right-hand side of the above equation is given by the correlation function $C^{i}{ }_{j}{ }^{i}{ }_{j}$. Thus, the dimensionless gravitational wave power spectrum is given by this correlation function. Therefore, from (19) one can calculate the dimensionless power spectrum for $h_{k}^{ \pm}$, where $h_{k}^{ \pm}$is the amplitude of either of the two gravitational wave polarization modes. Dropping the superscript \pm (due to symmetry both polarization modes will be equally excited) we obtain

$$
k^{3}|h(k)|^{2} \sim k^{-4}(8 \pi G)^{2} C^{i}{ }_{j}{ }_{j} .
$$

Inserting our result (5) for the correlation function yields

$$
k^{3}|h(k)|^{2} \sim \frac{64 \pi^{2} G^{2} T}{\ell_{s}^{3}}\left(1-T / T_{H}\right) \ln ^{2}\left[\frac{1}{\ell_{s}^{2} k^{2}}\left(1-T / T_{H}\right)\right],
$$


which, for temperatures close to the Hagedorn value reduces to

$$
k^{3}|h(k)|^{2} \sim\left(\frac{\ell_{P l}}{\ell_{s}}\right)^{4}\left(1-T / T_{H}\right) \ln ^{2}\left[\frac{1}{\ell_{s}^{2} k^{2}}\left(1-T / T_{H}\right)\right] .
$$

This shows that the spectrum of tensor modes is - to a first approximation - scale-invariant.

Discussion. Our result (22) for the power spectrum of gravitational waves should be compared to the result for the power spectrum of scalar metric fluctuations computed in [9]:

$$
\mathcal{P}_{\Phi}(k) \sim\left(\frac{\ell_{p l}}{\ell_{s}}\right)^{4} \frac{1}{1-T / T_{H}} .
$$

Note that for a fixed scale $k$, both (23) and (22) must be evaluated at the time $t_{i}(k)$ when the mode $k$ exits the Hubble radius at the end of the Hagedorn phase. Since $t_{i}(k)$ increases slightly as $k$ increases, the temperature $T\left(t_{i}(k)\right)$ will be slowly decreasing. Hence, the expression in front of the logarithm in our final expression (22) for the power spectrum of tensor fluctuations yields a slight blue tilt. Values of $k$ for which the perturbative analysis of string gas cosmology is consistent are on the high $k$ side of the zero of the logarithm in (22) - this follows from the condition $\left|\delta \Omega_{(1)}\right| \ll 1$ and (12). Hence, the logarithm factor in (22) adds to the blue tilt of the spectrum.

A heuristic way of understanding the origin of the slight blue tilt in the spectrum of tensor modes is as follows. The closer we get to the Hagedorn temperature, the more the thermal bath is dominated by long string states, and thus the smaller the pressure will be compared to the pressure of a pure radiation bath. Since the pressure terms (strictly speaking the anisotropic pressure terms) in the energy-momentum tensor are responsible for the tensor modes, we conclude that the smaller the value of the wavenumber $k$ (and thus the higher the temperature $T\left(t_{i}(k)\right)$ when the mode exits the Hubble radius, the lower the amplitude of the tensor modes. In contrast, the scalar modes are determined by the energy density, which increases at $T\left(t_{i}(k)\right)$ as $k$ decreases, leading to a slight red tilt.

It is also interesting to consider the tensor to scalar ratio $r$. Comparing (22) and (23) we see that this ratio, evaluated on a scale $k$ is given by

$$
r \sim\left(1-T\left(t_{i}(k)\right) / T_{H}\right)^{2} \ln ^{2}\left[\frac{1}{\ell_{s}^{2} k^{2}}\left(1-T\left(t_{i}(k)\right) / T_{H}\right)\right] .
$$

In principle (if the dynamical evolution from the Hagedorn phase to the radiation-dominated FRW phase were under complete analytical control) this quantity is calculable. If the string length were known, the factor $\left(1-T / T_{H}\right)$ could be determined from the normalization of the power spectrum of scalar metric fluctuations. Since the string length is expected to be a couple of orders larger than the Planck length, the above factor does not need to be very small. Thus, generically we seem to predict a ratio $r$ larger than in simple roll inflationary models.

Based on the results of this Letter it thus appears promising that our scenario will give rise to testable predictions.

The work of R.B. is supported by funds from McGill University, by an NSERC Discovery Grant and by the Canada Research Chairs program. The work of A.N. and C.V. is supported in part by NSF grant PHY-0244821 and DMS-0244464.

* email: rhb@hep.physics.mcgill.ca

$\dagger$ email: nayeri@schwinger.harvard.edu

‡ email: patil@hep.physics.mcgill.ca

$\S$ email: vafa@physics.harvard.edu

[1] R. H. Brandenberger and C. Vafa, "Superstrings In The Early Universe," Nucl. Phys. B 316, 391 (1989).

[2] A. A. Tseytlin and C. Vafa, "Elements of string cosmology," Nucl. Phys. B 372, 443 (1992) arXiv:hep-th/9109048.

[3] J. Kripfganz and H. Perlt, Class. Quant. Grav. 5, 453 (1988).

[4] R. H. Brandenberger, "Moduli stabilization in string gas cosmology," arXiv:hep-th/0509159

[5] R. H. Brandenberger, "Challenges for string gas cosmology," arXiv:hep-th/0509099

[6] T. Battefeld and S. Watson, "String gas cosmology," arXiv:hep-th/0510022

[7] R. Hagedorn, "Statistical Thermodynamics Of Strong Interactions At High-Energies," Nuovo Cim. Suppl. 3, 147 (1965).

[8] G. Veneziano, Phys. Lett. B 265, 287 (1991).

[9] A. Nayeri, R. H. Brandenberger and C. Vafa, "Producing a scale-invariant spectrum of perturbations in a Hagedorn phase of string cosmology," arXiv:hep-th/0511140

[10] A. Nayeri, in preparation

[11] R. H. Brandenberger, "Lectures on the theory of cosmological perturbations," Lect. Notes Phys. 646, 127 (2004) arXiv:hep-th/0306071.

[12] V. F. Mukhanov, H. A. Feldman and R. H. Brandenberger, "Theory Of Cosmological Perturbations. Part 1. Classical Perturbations. Part 2. Quantum Theory Of Perturbations. Part 3. Extensions," Phys. Rept. 215, 203 (1992).

[13] R. Brandenberger and N. Shuhmaher, "The Confining Heterotic Brane Gas: A Non-Inflationary Solution to the Entropy and Horizon Problems of Standard Cosmology," arXiv:hep-th/0511299

[14] R. Easther, B. R. Greene, M. G. Jackson and D. Kabat, "String windings in the early universe," JCAP 0502, 009 (2005) arXiv:hep-th/0409121.

[15] R. Danos, A. R. Frey and A. Mazumdar, "Interaction rates in string gas cosmology," Phys. Rev. D 70, 106010 (2004) arXiv:hep-th/0409162.

[16] S. Watson and R. Brandenberger, "Stabilization of extra dimensions at tree level," JCAP 0311, 008 (2003) arXiv:hep-th/0307044.

[17] S. P. Patil and R. Brandenberger, "Radion stabilization 
by stringy effects in general relativity," Phys. Rev. D 71, 103522 (2005) arXiv:hep-th/0401037.

[18] S. P. Patil and R. H. Brandenberger, "The cosmology of massless string modes," arXiv:hep-th/0502069

[19] S. P. Patil, "Moduli (dilaton, volume and shape) stabilization via massless F and D string modes," arXiv:hep-th/0504145

[20] R. Brandenberger, Y. K. Cheung and S. Watson, "Moduli stabilization with string gases and fluxes," arXiv:hep-th/0501032

[21] S. Watson, "Moduli stabilization with the string Higgs effect," Phys. Rev. D 70, 066005 (2004)
arXiv:hep-th/0404177.

[22] N. Deo, S. Jain, O. Narayan and C. I. Tan, Phys. Rev. D 45, 3641 (1992).

[23] N. Deo, S. Jain and C. I. Tan, Phys. Lett. B 220, 125 (1989).

[24] N. Deo, S. Jain and C. I. Tan, Phys. Rev. D 40, 2626 (1989).

[25] M. J. Bowick, "Finite temperature strings," arXiv:hep-th/9210016

[26] D. Mitchell and N. Turok, Nucl. Phys. B 294, 1138 (1987). 